\title{
High-fidelity is not superior to low-fidelity medical simulation and leads to overconfidence in medical students
}

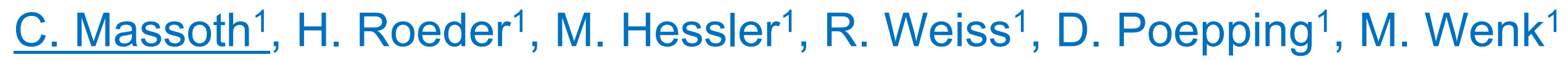 \\ Münster \\ 1Department of Anesthesiology and Intensive Care and Pain Medicine, University Hospital Muenster - Muenster (Germany)
}

Universitätsklinikum

\section{Background}

Simulation has become integral to the training of both undergraduate medical students as well as professionals.

Due to the increasing degree of realism and range of features, the latest mannequins are referred to as high-fidelity simulators.

\section{Objective}

To assess

-whether increased realism leads to a likewise improvement in trainees' outcome

-the influence on individual confidence and self-assessment

\section{Methods}

Ethics Committee approval: 2014-544-f-S

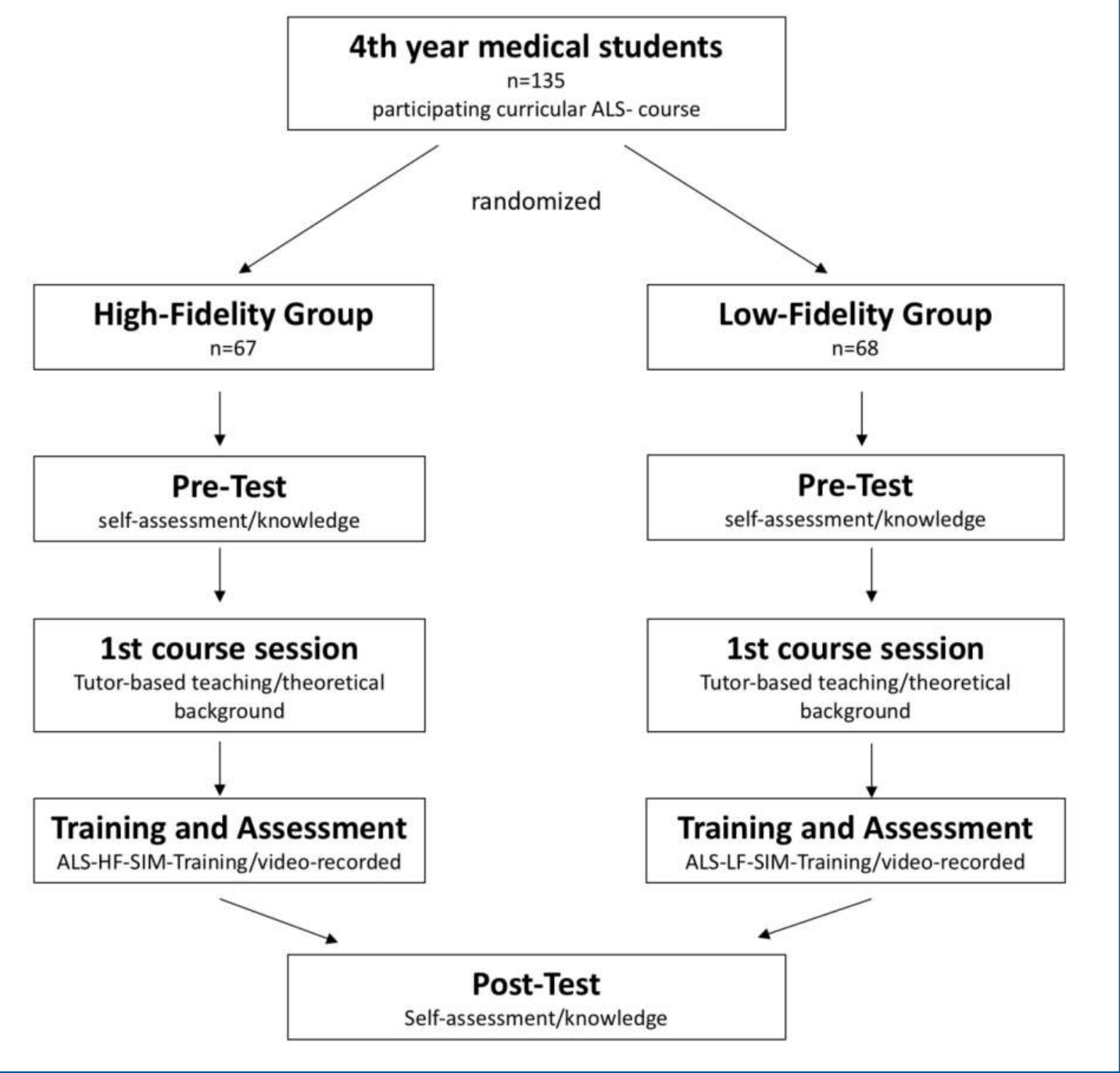

\section{Results}

Multiple-Choice knowledge test

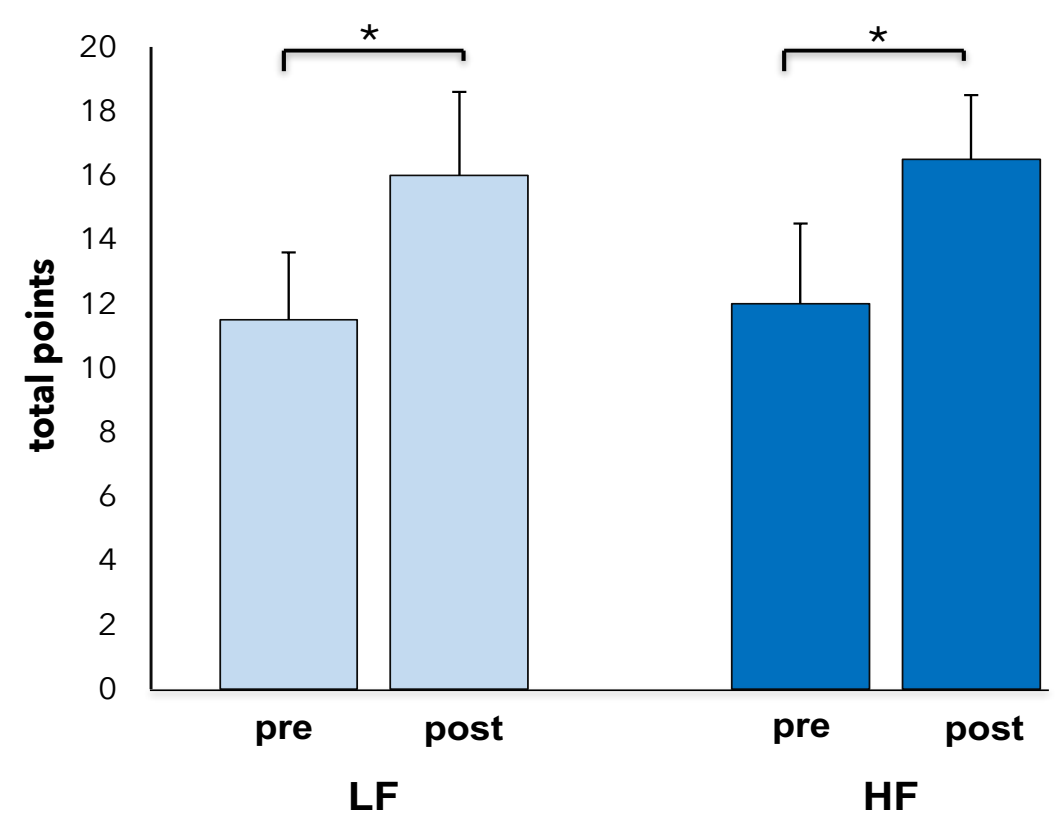

Fig. 1: Both groups improved their theoretical knowledge significantly $(p<0.001)$. No significant intergroup differences were detected $(p>0.05)$

\section{Video analysis}

Scoring of practical performance in 25 items resulted in comparable findings for both groups for most evaluation criteria. With regard to 4 items students in the LF group performed significantly better $(p<0.05)$.

\section{Self-assessment}

Participants of the high-fidelity group considered themselves to be advantaged, solely based on their group allocation, compared with those in the lowfidelity group, at both pre- and post-self-assessments. Selfrated assessment of their individual performance was also significantly overrated.

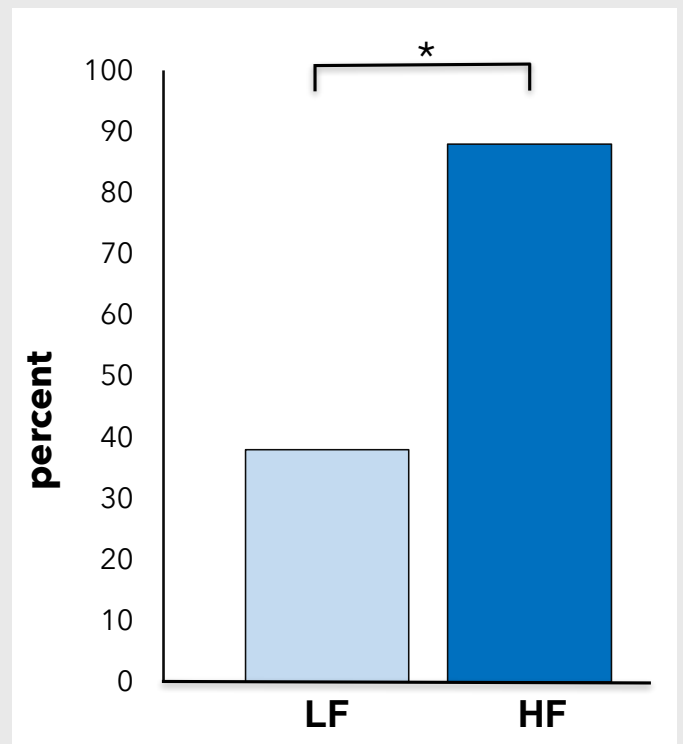

Fig. 2: Assessment of HF-Simulation as superior learning tool after the course $(p<0.01)$

\section{Conclusions}

As compared to LF-Simulation, the use of HF-Simulation:

$$
\begin{aligned}
& \rightarrow \text { led to equal or even worse performance and growth in knowledge } \\
& \rightarrow \text { induced undesirable effects as overrating the individual performance } \\
& \rightarrow \text { turned out as a disadvantageous learning tool in this scenario }
\end{aligned}
$$

